\title{
Determinación de marcadores inflamatorios y anabólicos articulares en caballos jóvenes estabulados con osteocondrosis tarsocrural
}

\section{Determination of inflammatory and anabolic joint markers in stabled young horses with tarsocrural ostechondrosis}

\author{
Jorge Carmona, ${ }^{1 *}$ Ph.D, Catalina López, ${ }^{1}$ MVZ, Marta Prades, ${ }^{2}$ Ph.D.
}

\begin{abstract}
${ }^{1}$ Universidad de Caldas, Departamento de Salud Animal, Grupo de Investigación Terapia Regenerativa, Calle 65 No 26-10, Manizales, Colombia. ${ }^{2}$ Universidad Autónoma de Barcelona, Departamento de Medicina y Cirugía Animal, Facultad de Veterinaria, Edificio V, Campus UAB. 08193 Cerdanyola del Vallés, España. *Correspondencia: carmona@ucaldas.edu.co.
\end{abstract}

Recibido: Junio 17 de 2009; Aceptado: Noviembre 24 de 2009.

\section{RESUMEN}

Objetivo. Describir los valores de las concentraciones en líquido sinovial (LS) de prostaglandina E2 (PGE2), óxido nítrico (NO), ácido hialurónico (HA) y proteína total (PT) en caballos jóvenes estabulados con osteocondrosis (OC) tarsocrural y correlacionar los valores de estos marcadores con el recuento total de células del LS, el grado de efusión sinovial (ES) y con el grado de cojera (GC). Materiales y métodos. Las concentraciones en LS de PGE2 fueron determinadas mediante ELISA, las de NO fueron valoradas por la reacción de Greiss, las de HA fueron medidas con radioinmunoanálisis RIA y las de PT mediante refractometría. Resultados. Once caballos entre 8 y 36 meses fueron incluidos. La mediana y el rango (R) de los grados de ES y GC fueron de 1.5 (R: 0.5-2) y 0 (R: 0-2), respectivamente. La mediana de la concentración de PGE2 y NO, respectivamente fue $130.6 \mathrm{pg} / \mathrm{mL}$ (R: $41-231.7 \mathrm{pg} / \mathrm{mL}$ ) y 2.92 mM (R: 1.3-6.3 mM).. La concentración de HA presentó una mediana de $312.4 \mathrm{mg} / \mathrm{mL}$ (R: $70-543.5 \mathrm{mg} / \mathrm{mL}$ ). La concentración promedio de PT fue de $1.2 \pm-0.36 \mathrm{~g} / \mathrm{dL}$. Se observó correlación negativa estadísticamente significativa entre las concentraciones sinoviales de HA y el grado de efusión sinovial $(\tilde{n}=-0.7 ; p=0.048)$ y el grado de cojera $(\tilde{n}=-0.78 ; p=0.014)$. Conclusiones. La concentración de HA en LS de caballos con OC podría estar negativamente correlacionada con la gravedad de los signos clínicos.

Palabras clave: Equinos, osteocondrosis, biomarcadores articulares. 


\section{ABSTRACT}

Objective. To describe the concentrations in synovial fluid (SF) of prostaglandin E2 (PGE2), nitric oxide (NO), hyaluronic acid (HA) and total protein (TP) in stabled young horses with tarsocrural OC and correlate the values of these markers with the SF total cell count the degree of synovial effusion (SE) and the degree of lameness (LD). Materials and methods. A PGE2 SF concentration was determined by ELISA, NO was determined by the Greiss reaction, HA was measured by RIA and TP by refractometry. Results. Eleven horses between 8-36 months were included. The median grades of SE and LD were 1.5 (range (R): 0.5-2) and 0 (R: 0-2), respectively. Median value for PGE2 was $130.6 \mathrm{pg} / \mathrm{mL}$ (R: $41-231.7 \mathrm{pg} / \mathrm{mL}$ ). Median value for NO was $2.92 \mathrm{mM}$ (R: $1.3-6.3 \mathrm{mM}$ ). Median value for HA was $312.4 \mathrm{mg} / \mathrm{mL}$ ( $R$ : $70-543.5 \mathrm{mg} / \mathrm{mL}$ ). The average concentration for TP was $1.2 \pm-0.36 \mathrm{~g} / \mathrm{dL}$. Statistically significant negative correlations between the concentrations of HA in synovial fluid and the degree of synovial effusion ( $\tilde{n}=-0.7, p=0.048$ ) and degree of lameness $(\tilde{n}=-0.78, p=$ $0.014)$ were observed. Conclusions. The concentration of HA in LS of horses with OC may be negatively correlated with the severity of clinical symptoms.

Key words: Equines, osteochondrosis, joint biomarkers.

\section{INTRODUCCIÓN}

La osteocondrosis (OC) es una enfermedad multifactorial con un componente hereditario, clasificada dentro del complejo de enfermedades ortopédicas del desarrollo en el caballo. La OC se desencadena por una deficiencia en la osificación endocondral del complejo epifisario-articular. Las articulaciones más afectadas son la tarsocrural, femoropatelar y metacarpofalangiana (1). La incidencia de la enfermedad puede oscilar entre $10-70 \%$ (2). La OC causa grandes pérdidas económicas a los productores, representadas por la devaluación del potencial atlético y reproductivo de los equinos afectados (3).

Clínicamente, las articulaciones con OC pueden presentar diferentes grados de distensión articular (efusión sinovial). La presencia de cojera no es típica en caballos con OC. Sin embargo, puede aparecer cuando los caballos jóvenes inician su entrenamiento (1). La cojera puede manifestarse sobre todo cuando las lesiones osteocondrales están ubicadas en sitios de gran movilidad y carga articular como la cresta intermedia de la tibia $(1,4)$. La OC siempre deberá ser descartada en potros que presenten distensión sinovial leve de tarsos, menudillos o babillas $(1,2,4)$.
Las articulaciones afectadas por OC pueden presentar fragmentos osteocondrales libres, exposición focalizada de hueso subcondral y sinovitis $(1,4)$. Este cuadro cursa con alteraciones bioquímicas inflamatorias del ambiente articular que perpetúan la inflamación de la membrana sinovial con la consecuente destrucción del cartílago articular sano (5-7). Agentes proinflamatorios primarios, como la interleucina 1 (IL-1), activan la expresión del sistema enzimático inflamatorio que incluye, entre otras, la expresión de la ciclooxigensa 2 (COX-2), y la sintetasa inducible de óxido nítrico (iNOS) (8). Estas enzimas a su vez producen prostaglandina E2 $\left(\mathrm{PGE}_{2}\right)$ y óxido nítrico (NO). Ambos metabolitos inflamatorios están asociados con dolor y efusión articulares y con el potencial desarrollo de osteoartritis (OA) (8) de la articulación inicialmente afectada con OC (1), si la causa incitante no es controlada (8).

El conocimiento del comportamiento de estos marcadores podría ser de utilidad para ampliar el conocimiento molecular de la OC y para ser usados como posibles indicadores de respuesta bioquímica frente a las intervenciones clínicas o quirúrgicas 
que se hagan en caballos con la enfermedad.

El ácido hialurónico (HA) es un componente fundamental del líquido sinovial (LS). Es un glicosaminoglicano no sulfatado, producido por las células sinoviales (9), pero también es producido por los diferentes tipos de tejidos conectivos en todo el cuerpo. La principal acción del HA en las articulaciones es promover su lubricación (10). A nivel sinovial el HA inhibe la migración de leucocitos, actúa como un cazador de radicales libres y ofrece condroprotección, ya que aumenta la síntesis de proteoglicanos $(9,11)$.

Los objetivos de esta investigación fueron 1) describir los valores de las concentraciones en LS de $\mathrm{PGE}_{2}$, NO (marcadores inflamatorios), HA (marcador de anabolismo articular) y proteína total en caballos jóvenes estabulados con OC tarsocrural y 2) correlacionar los valores de estos marcadores con el recuento total de células del LS, el grado de efusión sinovial y con el grado de cojera.

\section{MATERIALES Y MÉTODOS}

Sitio de estudio. El trabajo fue aprobado por las directivas del Hospital Veterinario . Los dueños de los caballos firmaron una hoja de autorización en la que se informaba de la naturaleza del estudio y de las posibles complicaciones derivadas de la punción articular. Los caballos evaluados en este estudio hacían parte de criaderos con condiciones ambientales, nutricionales y de manejo muy parecidas. Los caballos fueron evaluados clínicamente en sus criaderos de origen, por solicitud de los propietarios a causa de la distensión articular observada en los pacientes.

Criterios de inclusión en el estudio. El criterio para incluir un caballo en el estudio obedeció a tres factores: a) edad (menores de 3 años), b) presencia unilateral visible de fragmentos osteocondrales en la articulación tarsoclural, acompañados de efusión sinovial y c) los potros no deberían estar en entrenamiento y deberían estar estabulados.

Diseño del estudio. Los caballos incluidos fueron evaluados mediante exámenes físico general y locomotor, pruebas complementarias (química clínica), estudio radiológico y determinación bioquímica de las concentraciones en LS de $\mathrm{PGE}_{2}$, NO y HA.

Examen clínico y del aparato locomotor. Cada caballo fue evaluado para incluir animales libres de enfermedad con manifestaciones clínicas, para ello adicionalmente se realizó un hemograma y un panel bioquímico general (glucosa, AST, creatinina y albumina). Posteriormente, se realizó evaluación del aparato locomotor. Los caballos que presentaban efusión sinovial unilateral del tarso fueron sometidos a un estudio radiológico de esa misma región anatómica.

Calificación del grado de efusión sinovial (distención articular). Se realizó evaluación semicualitativa del grado de efusión sinovial en cada caballo (12-14) para esto se combinaron los resultados de las observaciones clínicas y los hallazgos ultrasonográficos de cada articulación evaluada. La evaluación clínica incluyó el aspecto externo de la articulación afectada en comparación con la articulación contralateral sana, respecto a la temperatura, textura de la cápsula, grado de movimiento articular, grado de dolor durante la palpación y movimiento pasivo, entre otros. El examen ecográfico se realizó con un equipo Acuson-Aspen (1230 Shorebird Way, Mountain View CA, USA). El examen fue realizado con una sonda lineal de $10 \mathrm{MHz}$ e incluyó: evaluación de la superficie articular, cantidad de líquido sinovial, grosor y aspecto de la membrana sinovial y sus vellosidades y presencia de fragmentos osteocondrales. El grado de efusión sinovial (distensión articular) fue calificado en una escala de 0 a 3 , donde $0=$ Articulación de aspecto normal. 1 =efusión levemente palpable, sin engrosamiento de la cápsula articular. 2=Efusión palpable, con engrosamiento capsular moderado y una reacción dolorosa leve durante la palpación profunda. 3=Efusión severa, con la membrana 
sinovial engrosada, presencia de fragmentos osteocondrales y reacción dolorosa durante la palpación articular y su movilización pasiva (12). Las medidas ultrasonográficas fueron realizadas siempre en el mismo punto anatómico (receso dorsolateral medial inmediatamente por debajo del maléolo medial de la tibia en cada paciente y por el mismo clínico).

Calificación del grado de cojera. El grado de cojera fue calificado de 0-5, según los parámetros establecidos por la AAEP (13), aunque la asignación de grados medios (0.5) también fue permitida. Solamente, se consideró el trote en línea recta sobre una superficie dura.

Examen radiológico. El tarso de cada caballo con efusión sinovial fue evaluado mediante las 4 proyecciones estándar de esa articulación (2).

Determinaciones bioquímicas en líquido sinovial. Cada articulación afectada fue preparada asépticamente para realizar punción articular, previa sedación. Una muestra de líquido sinovial de $1 \mathrm{ml}$ fue depositada en un tubo con EDTA para análisis citológico (recuento celular y determinación de proteína total). Otra muestra de $1 \mathrm{ml}$ fue depositada en un tubo con heparina de litio. La muestra fue inmediatamente centrifugada a $1780 \times \mathrm{g}$ durante 5 minutos y seguidamente se extrajo el líquido sinovial (aproximadamente $0.7 \mathrm{ml}$ ) libre de sus células, el cual fue depositado en tubos tipo eppendorf y transportados en una nevera con hielo hasta el laboratorio. Las muestras fueron congeladas a $-80^{\circ} \mathrm{C}$.

Determinación de la $\mathbf{P G E}_{2}$ en el líquido sinovial. Las concentraciones de $\mathrm{PGE}_{2}$ en LS fueron determinadas mediante una prueba de ELISA en sándwich construida con anticuerpos comerciales (Quantiquine, R\&D, Systems, UK) para seres humanos. La estructura de este eicosanoide está muy conservada entre especies y no existe reactividad cruzada de esta molécula con otros eicosanoides, por tal razón puede ser usada para medir $\mathrm{PGE}_{2}$ en el caballo. El ensayo fue realizado de acuerdo con las instrucciones del fabricante. La lectura de absorbancia fue realizada a $405 \mathrm{~nm}$ mediante un lector de ELISA. La detección límite del ensayo fue de $15 \mathrm{pg} / \mathrm{ml}(14,15)$.

Determinación del NO en el líquido sinovial. Las concentraciones de NO en las muestras de líquido sinovial fueron determinadas a través de su producto de degradación, los nitritos $\left(\mathrm{NO}_{2}\right)$, por medio del ensayo de diazotización (reacción de Greiss), previa reducción de los nitratos $(16,17)$. El $\mathrm{NO}_{2}$ es un producto final y estable del $\mathrm{NO}$ en una cantidad aproximada de $51.5 \pm 3.5 \%$ del total ON producido (14).

Se construyó una recta patrón a partir de un estándar de nitrito sódico al 100 mM. La recta fue iniciada a partir de una dilución 1/1000 del estándar con una concentración máxima de $100 \mathrm{mM}$. Las muestras fueron centrifugadas a $1780 \times \mathrm{g}$ durante $10 \mathrm{~min}$ y sin diluir fueron colocadas en alícuotas de $100 \mathrm{ml}$ en placas de 96 pozuelos. El contenido de cada pozo fue mezclado con $100 \mathrm{ml}$ de una solución de trabajo fabricada a partir de 2 (dos) partes iguales de $3 \mathrm{ml}$ de ácido fosfórico al 5\%, $51 \mathrm{ml}$ de agua y sulfanilamida al $1 \%(0.5 \mathrm{~g} / 50 \mathrm{ml}$ de ácido fosfórico al $5 \%)$ y N-1-napthilethileno diamina (NEDA) al $0.1 \%$ ( $0.05 \mathrm{~g}$ en $50 \mathrm{ml}$ agua). Cada muestra permaneció 10 min bajo incubación a temperatura ambiente y posteriormente fue medida en un lector de ELISA a $540 \mathrm{~nm}$. La sensibilidad del método fue de $1 \mathrm{mM}$. Todos los resultados se expresaron como mM (17).

Determinación de ácido hialurónico en el líquido sinovial. EI HA fue determinado mediante radioinmunoanálisis (RIA), con un kit comercial (Pharmacia, HA Test, Pharmacia \& Upjohn Diagnostics AB, Uppsala, Sweden). La prueba se basó en la mezcla de $200 \mathrm{ml}$ de proteínas de unión de ácido hialurónico aislada de cartílago bovino (HABP-125) con $100 \mathrm{ml}$ de muestra de LS. Luego de una incubación de 60 minutos a $4-20^{\circ} \mathrm{C}$, se agregó $100 \mathrm{ml}$ de AH-sefarosa y se esperó una incubación adicional de 45 min a la misma temperatura que el anterior. Luego se agregaron $2 \mathrm{ml}$ de solución de decantación y se centrifugó la muestra durante 10 minutos a $600 \mathrm{xg}$. Los niveles de HA de las muestras fueron medidas mediante un contador gamma. El principio de esta prueba, 
consiste en cuantificar la HABP-125 no unida al ácido hialurónico. De esta manera la radioactividad medida es inversamente proporcional a la cantidad de ácido hialurónico presente en la muestra $(18,19)$.

Análisis estadístico. Los datos obtenidos fueron analizados con el programa estadístico SPSS 16.0 (SPSS inc., IL, USA). Los valores de todas las variables evaluadas, a excepción de los niveles de proteína total del fluido sinovial, fueron analizados mediante pruebas no paramétricas, ya que estos datos presentaron una distribución no normal (prueba de Shaphiro-Wilk $\mathrm{p}<0.05$ ) y las transformaciones logarítmicas (LogY) y de arcoseno no permitieron normalizar los datos. Se hizo un análisis de correlaciones de Spearman (ñ) entre las variables bioquímicas y clínicas evaluadas. Un valor de $p<0.05$ fue aceptado como estadísticamente significativo para esta prueba. Todos los resultados fueron expresados como medianas (rangos) a excepción de los valores de proteína total el LS, que fueron presentados como promedio (desviación estándar).

\section{RESULTADOS}

Once caballos raza pura español alcanzaron los criterios de inclusión del estudio. De ellos 10 fueron machos y 1 fue hembra, con un rango de edad comprendido entre 8-36 meses. El estudio radiológico reveló que 10 caballos presentaban un fragmento osteocondral en la cresta intermedia de la tibia y 1 presentaba un fragmento en el labio lateral de la tróclea del astrágalo. Los caballos incluidos presentaban efusión sinovial con un rango de calificación de 0.5-3 (mediana 1.5). Seis de los 11 caballos no presentaban cojera, los cinco restantes presentaban un grado de cojera que oscilaba en un rango de $0.5-2$ grados. La mediana general del grado de cojera en los pacientes seleccionados fue de 0 (rango 0-2).

El análisis citológico del líquido sinovial presentó recuento celular mediano de 150 células/mL (rango: 50-200 células/ $\mathrm{mL}$ ), caracterizado por células mononucleares poco reactivas. Los caballos incluidos presentaron un nivel medio de proteínas totales en líquido sinovial de $1.2 \pm-0.36 \mathrm{~g} / \mathrm{dL}$. Las concentraciones medianas de $\mathrm{NO}$ en líquido sinovial fueron de $2.92 \mathrm{mM}$ (rango 1.33-6.27 mM). La $\mathrm{PGE}_{2}$ presentó niveles medianos en el líquido sinovial de 130.6 $\mathrm{pg} / \mathrm{mL}$ (rango 41-231.7 pg/mL). EI HA presentó concentraciones sinoviales medianas de $312.4 \mathrm{mg} / \mathrm{mL}$ (rango $70-$ $543.5 \mathrm{mg} / \mathrm{mL}$ ).

Se observó correlación negativa estadísticamente significativa entre las concentraciones sinoviales de $\mathrm{HA}$ y el grado de efusión sinovial $(\tilde{n}=-0.7 ; p=0.048)$ (Figura 1) y entre las concentraciones sinoviales de HA y el grado de cojera ( $\tilde{n}=-0.78$; $\mathrm{p}=0.014$ ) (Figura 2). No se hallaron correlaciones entre los marcadores

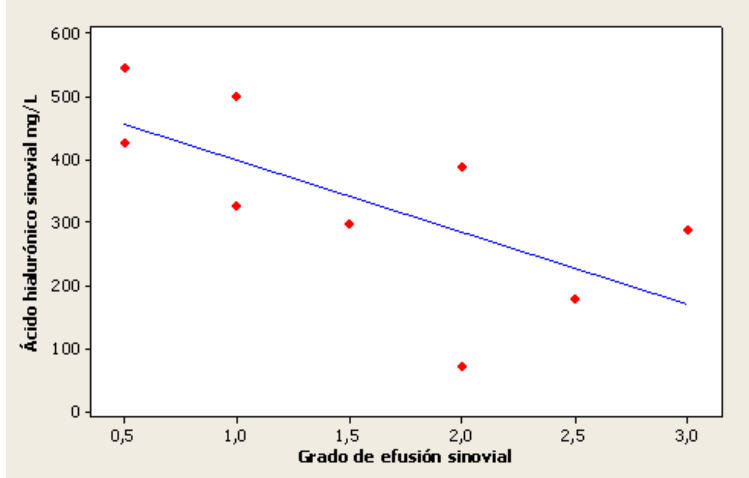

Figura 1. Gráfico de dispersión sobre la concentración de ácido hialurónico en líquido sinovial Vs el grado de efusión sinovial en los caballos del estudio.

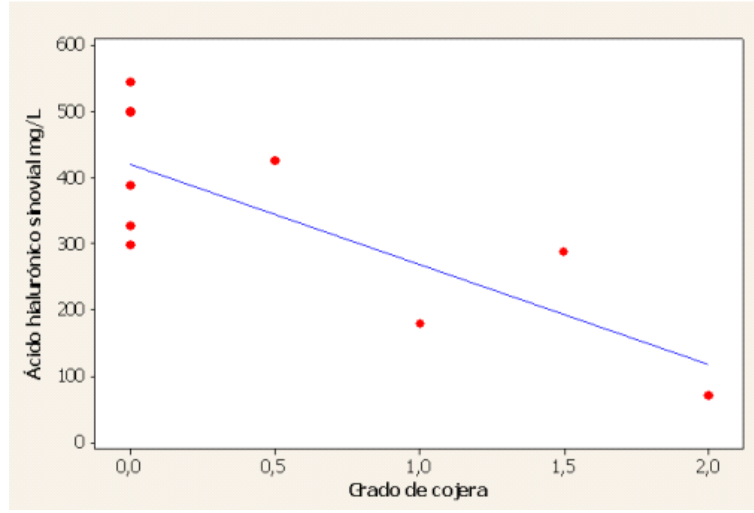

Figura 2. Gráfico de dispersión sobre la concentración de ácido hialurónico en líquido sinovial Vs el grado de cojera en los caballos del estudio. 
inflamatorios, los recuentos celulares del líquido sinovial, las concentraciones sinoviales de proteína total y los grados de efusión sinovial o de cojera.

\section{DISCUSIÓN}

Investigaciones recientes en caballos jóvenes con OC tarsocrural implicaron a los eicosanoides (LTB4 y PGE 2 ) (5) y radicales libres como el NO (6) como protagonistas de la génesis y perpetuación de la efusión sinovial y de los daños del cartílago articular de las articulaciones afectadas por esa enfermedad $(5,6)$. de Grauw et al $(5)$ reportaron concentraciones significativamente más elevadas de $\mathrm{PGE}_{2}$ en el líquido sinovial en caballos con OC tarsocrural (362.3 $293.6 \mathrm{pg} / \mathrm{mL})$ en comparación con el líquido sinovial de las articulaciones similares en caballos sanos $(127.3 \pm 33.6 \mathrm{pg} / \mathrm{mL})$. En el estudio presentado se encontraron concentraciones medianas más bajas de $\mathrm{PGE}_{2}$ en LS (130.6 pg/mL; rango: 41-231.7 $\mathrm{pg} / \mathrm{mL}$ ) en comparación con los resultados descritos por de Grauw et al (5) para los caballos con OC tarsocrural y valores muy similares a los de los caballos del grupo control de ese mismo estudio.

Es importante señalar que el equipo de la presente investigación y el de Grauw et al (5) utilizaron anticuerpos contra $\mathrm{PGE}_{2}$ del mismo fabricante (R\&D Systems). La disparidad observada entre ambos estudios pudo estar relacionada, entre otras, con i) la metodología utilizada para recoger y procesar el líquido sinovial y ii) con los diferentes regímenes de ejercicio de los caballos en cada estudio (20). En el presente trabajo se utilizaron tubos con heparina-litio para recoger y procesar las muestras de líquido sinovial, mientras que de Grauw et al (5) utilizaron tubos sin anticoagulante y además esperaron 1 hora para realizar el centrifugado de líquido sinovial. Cuando los leucocitos entran en contacto con una superficie poco habitual (en este caso la pared del tubo de recolección del LS), aumentan su actividad metabólica inflamatoria (19). Además, la heparina tiene la capacidad de prevenir este tipo de reacción inflamatoria (19).
Por otro lado, Van Den Boom et al (20) encontraron que la concentración de $\mathrm{PGE}_{2}$ puede aumentar en el líquido sinovial de caballos sometidos a ejercicio en comparación con caballos sedentarios. Los caballos en el presente estudio estaban bajo estabulación para evitar el efecto del ejercicio sobre el aumento de este metabolito en el líquido sinovial (20).

Los niveles de HA en el líquido sinovial han sido documentados en caballos con articulaciones sanas (21-23) y en pacientes con diferentes artropatías, como osteoartritis (OA) (23), artritis traumática (24), enfermedad navicular (25) fragmentación osteocondral traumática (26) y artritis infecciosa (24), entre otras. Sin embargo, en la literatura revisada, los autores no pudieron encontrar un reporte sobre las concentraciones de este marcador anabólico en caballos con OC tarsocrural. Los valores HA en LS de caballos sanos o con diferentes artropatías varían ostensiblemente en cada estudio, principalmente por el tipo de método empleado en la medición de este metabolito. La técnica (RIA) empleada para medir la concentración de HA en el LS de los caballos del presente estudio (19) no fue utilizada en los trabajos revisados en caballos. Una desventaja del presente trabajo radicó en que no se midieron las concentraciones sinoviales de HA en articulaciones sanas con la finalidad de comparar estos valores con los de las afectadas por OCD.

Los hallazgos más interesantes de la presente investigación fueron las correlaciones negativas observadas entre los niveles de HA en LS y los grados de efusión sinovial $(\tilde{n}=-0.7 ; p=0.048)$ y cojera $(\tilde{n}=-0.78 ; p=0.014)$. En caballos con OA se ha observado una disminución de las concentraciones en LS de HA respecto a controles sanos (24). La OA equina cursa frecuentemente con sinovitis, la cual produce efusión sinovial (8). Es posible que el aumento de los niveles del fluido sinovial de las articulaciones con OA produzca un efecto dilucional sobre este marcador (21). Quizás este mismo hecho pudo ocurrir en los caballos de este reporte. Por otra parte, es posible pensar que la membrana sinovial inflamada tiene una capacidad metabólica 
disminuida para producir HA (27), tal como fue evidenciada en este estudio.

Otro hecho llamativo fue que el grado de cojera y el grado de efusión sinovial no mostraron una correlación estadísticamente significativa en los caballos del presente reporte. Sin embargo, las concentraciones de $\mathrm{HA}$ en LS y no las de $\mathrm{PGE}_{2}$ se asociaron con el grado de cojera de los caballos de la presente investigación. Los resultados de este trabajo concuerdan con los resultados reportados por de Graw et al (5) para caballos con OC tarsocrural. Sin embargo, difieren para los hallazgos encontrados en caballos con otras artropatías $(28,29)$, en los que se observó una correlación significativa entre el dolor articular (cojera) y los niveles incrementados de este marcador. Posiblemente, el dolor articular en caballos con OC este más asociado con disminución de las concentraciones de HA en LS que con el aumento de los niveles de $\mathrm{PGE}_{2}$, tal como fue observado en el presente trabajo. Sin embargo, este hecho deberá ser comprobado en un estudio con controles que incluya un alto número de pacientes con OC. Los resultados observados en esta investigación podrían servir para confirmar que la administración oral $(28,29)$ de $\mathrm{HA} \mathrm{a}$ caballos con OC podría ayudar a disminuir la sintomatología clínica asociada con la enfermedad $(29,30)$. Sin embargo, es necesario desarrollar una investigación clínica doble ciego controlada que incluya un gran número de pacientes con gran homogeneidad y en los que se puedan medir marcadores sinoviales y plasmáticos (además del HA) que permitan esclarecer el mecanismo por el cual él HA produce disminución de la efusión sinovial (y posiblemente cojera) en caballos con OC.

Para concluir, esta investigación aporta nuevo conocimiento sobre marcadores inflamatorios y anabólicos en caballos con OC tarsocrural. La concentración de HA en LS de caballos con esa patología podría estar negativamente correlacionada con la gravedad de los signos clínicos en caballos. La administración oral de HA podría ser una estrategia para disminuir los signos clínicos asociados a la OC en el caballo.

\section{Agradecimientos}

Los autores agradecen a todas las personas que colaboraron con la ejecución técnica del proyecto: Antoni Iborra, Laia Viñals, Paz Martínez, Ramón Deulofeu, Rafaela Cuenca, y Pau Serra. También, agradecen el apoyo de Bio-ibérica S.A, Barcelona, España, y muy especialmente a Daniel Martínez y Ana Ubia.

\section{REFERENCIAS}

1. McIlwraith CW. Clinical aspects of osteochondritis dissecans. In: McIlwraith CW, Trotter GW, eds. Joint disease in the horse. Philadelphia: WB Saunders Co, 1996; 369-374.

2. Wittwer $\mathrm{C}$, Hamann $\mathrm{H}$, Rosenberger $\mathrm{E}$, Distl $O$. Prevalence of osteochondrosis in the limb joints of South German Coldblood horses. J Vet Med A Physiol Pathol Clin Med 2006; 53: 531-539.

3. Jeffcot LB. Osteochondrosis: an international problem for the horse industry. J Equine Vet Scien 1996; 16: 32-37.
4. Van Weeren PR. Etiology, diagnosis and treatment of OC(D). Clin Tech Equine Pract 2006; 5: 248-258.

5. de Grauw JC, Brama PA, Wiemer P, Brommer $\mathrm{H}$, van de Lest $\mathrm{CH}$, van Weeren PR. Cartilage-derived biomarkers and lipid mediators of inflammation in horses with osteochondritis dissecans of the distal intermediate ridge of the tibia. Am J Vet Res 2006; 67: 1156-1162. 
6. Gangl $M$, Serteyn D, Lejeune JP, Schneider N, Grulke S, Peters F, et al. A type II-collagen derived peptide and its nitrated form as new markers of inflammation and cartilage degradation in equine osteochondral lesions. Res Vet Sci 2007;82:68-75.

7. Sutton S, Clutterbuck A, Harris P, Gent $\mathrm{T}$, Freeman $\mathrm{S}$, Foster $\mathrm{N}$, et al. The contribution of the synovium, synovial derived inflammatory cytokines and neuropeptides to the pathogenesis of osteoarthritis. Vet J 2009; 179:10-24.

8. Carmona JU, Prades, M. Pathophysiology of osteoarthritis. Comp Equine 2009; 4: $28-40$.

9. Howard RD, McIIwraith CW: Hyaluronan and its use in the treatment of equine joint disease. In McIIwraith CW, Trotter GW (eds). Joint disease in the horse. Philadelphia: WB Saunders Company; 1996.

10. Popot MA, Bonnaire J, Guéchot J, et al. Hyaluronan in horses: physiological production rate, plasma and synovial fluid concentrations in control conditions and following sodium hyaluronate administration. Equine Vet ] 2004; 36:482-487.

11. Frean SP, Abraham LA, Lees P: In vitro stimulation of equine articular cartilage proteoglycans synthesis by hyaluronan and carprofen. Res Vet Sci 1999; 67:181-188.

12. Carmona JU, Argüelles D, Climent F, Prades M. Autologous platelet concentrates as a treatment of horses with osteoarthritis: A preliminary pilot clinical study. J Equine Vet Scien 2007; 4: 167-170.

13. American association of equine practitioners (AAEP). Guide for veterinary service and judging of equestrian events: definition and classification of lameness. AAEP 1991.
14. Von Rechemberg B, McIlwraith CW, Akens MK, Frisbie DD, Leutenegger $C$, Auer JA. Spontaneous production of nitric oxide (NO), prostaglandin (PGE2), and neutral metalloproteinases (NMPs) in media of explant cultures of equine synovial membrane and articular cartilage from normal and osteoarthritic joints. Equine Vet J 2000; 32:140-150.

15. Bertone AL, Palmer JL. Synovial fluid cytokines and eicosanoids as markers of joint disease in horses. Vet Surg 2001; 30:528-538.

16. Archer S. Measurement of Nitric Oxide in biological models. FASEB J 1993; 7:340-360.

17. Carmona JU, D Argüelles, M Prades. Niveles de factor de crecimiento transformante beta-3 y óxido nítrico en cuatro concentrados autólogos de plaquetas y plasma derivados de sangre equina. Arch Med Vet 2008; 40: 155-160.

18. Guechot J, Laudat A, Loria A, Serfaty L, Poupon R, Giboudeau J. Diagnostic accuracy of hyaluronan and type III procollagen amino-terminal peptide serum assays as markers of liver fibrosis in chronic viral hepatitis $\mathrm{C}$ evaluated by ROC curve analysis. Clin Chem 1996; 42: 558-63.

19. Lappegård KT, Riesenfeld J, Brekke OL et al. Differential effect of heparin coating and complement inhibition on artificial surface-induced eicosanoid production. Ann Thorac Surg 2005; 79: 917-923.

20. Van den Boom $\mathrm{R}$, van de Lest $\mathrm{CH}$, Bull $S$, et al. Influence of repeated arthrocentesis and exercise on synovial fluid concentrations of nitric oxide, prostaglandin E2 and glycosaminoglycans in healthy equine joints. Equine Vet J 2005; 37: 250-256.

21. Viitanen M, Bird J, Maisi P, et al. Differences in the concentration of various synovial fluid constituents 
between the distal interphalangeal joint, the metacarpophalangeal joint and the navicular bursa in normal horses. Res Vet Sci 2000; 69:63-67

22. Popot MA, Bonnaire $Y$, Guéchot J, Toutain PL. Hyaluronan in horses: physiological production rate, plasma and synovial fluid concentrations in control conditions and following sodium hyaluronate administration. Equine Vet ] $2004 ; 36: 482-487$.

23. Taylor SE, Weaver MP, Pitsillides AA, Wheeler BT, Wheeler-Jones CP, Shaw DJ, Smith RK. Cartilage oligomeric matrix protein and hyaluronan levels in synovial fluid from horses with osteoarthritis of the tarsometatarsal joint compared to a control population. Equine Vet J 2006; 38:502-507.

24. Tulamo RM, Houttu J, Tupamäki A, Salonen $M$ Hyaluronate and large molecular weight proteoglycans in synovial fluid from horses with various arthritides. Am J Vet Res 1996; 57:932-7.

25. Viitanen M, Bird J, Makela O, Schramme M, Smith R, Tulamo RM, May S. Synovial fluid studies in navicular disease. Res Vet Sci 2001; 71:201-206.

26. Brown MP, Trumble TN, Plaas $A H$, Sandy JD, Romano M, Hernandez J, Merritt KA. Exercise and injury increase chondroitin sulfate chain length and decrease hyaluronan chain length in synovial fluid. Osteoarthritis Cartilage 2007; 15:1318-1325.
27. Pitsillides AA, Worrall JG, Wilkinson LS, Bayliss MT, Edwards JC. Hyaluronan concentration in non-inflamed and rheumatoid synovium. $\mathrm{Br}$ J Rheumatol 1994; 33:5-10.

28. Kawcak CE, Frisbie DD, Trotter GW, McIlwraith CW, Gillette SM, Powers BE, Walton RM. Effects of intravenous administration of sodium hyaluronate on carpal joints in exercising horses after arthroscopic surgery and osteochondral fragmentation. Am J Vet Res 1997;58:1132-40.

29. Martínez-Puig D, Carmona JU, Argüelles D, Deulofeu R, Ubia A, Prades $M$. Oral hyaluronic acid administration improves osteochondrosis clinical symptoms and slightly increses intraarticular concentration of hyaluronic acid in a horse model: a pilot survey [resumen]. Osteoarthritis Cartilage 2007; 15:C62c63.

30. Bergin BJ, Pierce SW, Bramlage LR, Stromberg A. Oral hyaluronan gel reduces post operative tarsocrural effusion in the yearling Thoroughbred. Equine Vet J 2006; 38: 375-8. 